\title{
Improvement of Hub Strategy in Robot Soccer Game
}

\author{
Jie Wu*, Václav Snášel*, Eliška Ochodková*, Jan Martinovič*, Václav Svaton* and Ajith Abraham ${ }^{\dagger}$ \\ * Department of Computer Science \\ VŠB - Technical University of Ostrava, Czech Republic \\ defermat2008@hotmail.com, \{vaclav.snasel,eliska.ochodkova,jan.martinovic,vaclav.svaton.st\}@vsb.cz \\ ${ }^{\dagger}$ Machine Intelligence Research Labs \\ Scientific Network for Innovation and Research Excellence, WA, USA \\ ajith.abraham@ieee.org
}

\begin{abstract}
The robot soccer game is full of challenging in the field of robot and artificial intelligence. Strategy is a kernel subsystem of robot soccer game. In our work, we present an approach to describe the strategies of the game, based on which a method to improve hub strategy is proposed. The experiment that eliminates the impact of external factors at mostly shows the effectiveness of strategic description and validity of our method.
\end{abstract}

Keywords-robot soccer; strategy; artificial intelligence;

\section{INTRODUCTION}

Soccer robotics combines artificial intelligence and mobile robotics with the popular sport of soccer. In essence, it studies how mobile robots can be built and trained to play a game of soccer. Many pure discipline areas, such as mathematics, physics, electronics, computing, etc, must be brought together into an integrated whole.

In robot soccer, the game situation in the playground is typically read in terms of the robots postures and the balls position. Using real-time information of this dynamically changing game situation, the system of robot soccer team would need to continually decide the action of each team robot, and to direct each robot to perform a selected action. On all accounts, the purpose is to get the team robots to exhibit some artificial form of cooperation. Naturally, good strategies are needed to decide the roles and actions of the team robots during the game.

In general games, a strategy refers to one of the options that a player can choose. A strategy must specify what action will happen in each contingent state of the game. For example, in robot soccer game, if the opponent robot does $\mathrm{A}$, then team robot takes action $\mathrm{B}$, whereas if the opponent robot does $\mathrm{C}$, team robot takes action $\mathrm{D}$. All strategies have a similar objective where the player could achieve the preferred target in order to defeat the opponent through a sequence of actions.

At present, few research is focused on the strategy of games. In these games, such as Chess, Checkers, Robot Soccer, and so on, the strategy implicates pivotal factors that determine victory or defeat in the game. However, in present technologies, the solutions of Chess or Checkers depend on search algorithms mostly [4], [8]. For robot soccer, the search technology is far from sufficient. We must research the strategy itself so that we can approach to the nature of intelligence.

Description of strategies is very important to strategies set, based on which we can discuss the performance of strategies set. Strategic description is an open question in the field of robot soccer game. The description of strategies should comprise all pivotal factors to the soccer game on strategic level. At the same time, the description should be succinct, and the strategies can be retrieved.

At present, there are many ways to describe strategy. Different description method implies different ideas of solution. For example, a decision tree of strategies is likely to lead to search technologies and recognition, a case of game situation would result in case retrieval. In our work, strategies could be described as a directed graph, by which we can observe the morphology of strategies set [9], such as isolated strategy, chain strategies, loop strategies, and so on. Morphology of strategies set is an important issue to strategic analysis. For example, in robot soccer game, the loop strategies are likely to make robots be in a trap of executing repeated actions. Hub strategy is another problem. The hub strategy links to many other strategies, it is hinge in the strategies set because many other strategies have to be achieved through it. It plays an important role to the followup game situation. Opponent may hinder the implementation of many strategies by attacking hub strategy. Therefore it is worth while to regard the problem of hub strategy.

This paper is organized as follows. Section II introduces the strategic description in our work. Based on the strategic description, in section III we propose a ranking model by which we could detect hub strategy. Section IV presents a method to improve the problem of hub strategy and prove its validity by simulation game of robot soccer. Finally, section $\mathrm{V}$ draws the conclusions.

\section{Description OF STRATEgies}

Description of Strategies is the basis of the robot soccer game. Many different forms of strategic description have been developed to support corresponding decision-making system. Bezek et al. [1], [2] propose a multi-agent strategic 
modeling of robot soccer. In their work, the strategy is described as a combination of agent roles, node positions and sequence of actions. Node positions correspond to agent positions in the game field that is divided into several areas, such as left-wing, center-of-the-field, right-wing, left-half, right-half, and so on. Huang and Liang [5] bring forward a strategy-based decision-making system for robot soccer game which implemented by a decision tree. In the tree, there are 12 strategies that are clustered by ball's position, possession of ball, team players, etc. In [7], a case is defined by $(P, A, K)$, where $P$ is the problem description, $A$, the solution description, and $K$, the case scope representation. The problem description corresponds to a set of features, including ball's global position, defending goal, teammates' global positions, and opponents' global positions, which describe the current world state. The solution of a case corresponds to the sequences of actions each teammate performs, such as "get the ball", "kick", or "pass ball to robot $t_{m i}$ ", and so on. All of these approaches mentioned above contain two common elements, i.e. grid positions and possession of ball. These common elements are also reflected in our description of strategies.

In our work, the game is separated into logical and physical parts [6]. The logical part includes the strategy selection, calculation of robot movement and adaptation of rules to the opponent's strategy. The physical part includes robot actual movement on the game field and recognition of the opponent movement. The logical part is independent of the physical part because we can calculate movements of the opponent robots as well as movements of own robots.

By separating the game into two parts, the logical part is independent of the field size and the resolution of the camera used in visual information system. In the logical part, the game is represented as an abstract grid with a very high resolution, which ensures a very precise position of robots and ball. However, this very detailed representation of the game field is not suitable for strategy description. Too many rules are required to describe robot behavior. Therefore, a strategy grid is used, which has a much lower resolution than an abstract grid. This simplification is sufficient because it is unnecessary to know the robots exact position. It is enough to know the robot's approximate position for strategy realization (see Fig. 1). We don't need to know the detailed position of the ball also, because in the strategy grid the tactic skill would lead robot to catch the ball. When the physical part is used, based on the game field size and camera resolution, we only need to transform the abstract grid into physical coordinates.

For the robot soccer game, the strategies can be treated as a series of actions under some certain conditions that may contain the information of position, velocity or acceleration, and so on. The position information, representing the situation of both sides, is much more important than the others.

Definition 1 (Description of A Strategy): According to

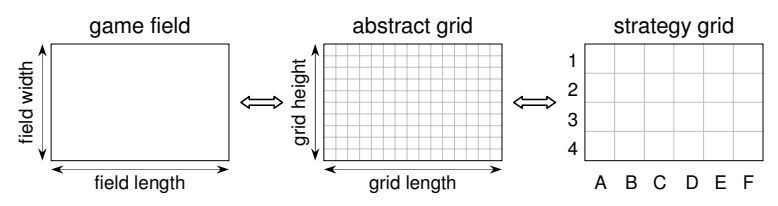

Figure 1. Inner game representation.

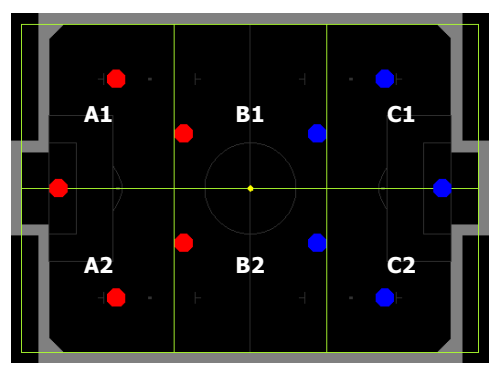

Figure 2. An example case.

strategy grid, the strategy can be expressed easily as $(M$, $O, B, D)$, where $M$ is the teammates' positions of mine, $O$, opponents' positions, $B$, ball position, and $D$, my teammates' destination grids.

Example 1 (A Strategy):

- Mine A1 A2 B1 B2;

- Oppo B1 B2 C1 C2 ;

- Ball B2 ;

o Dstn A1 B2 C1 C2 .

Example 1 shows a strategy stored in a file, which corresponds to the case shown in Fig. 2. It means "If $\left(M_{1}, M_{2}, M_{3}, M_{4}\right)$ is close to $(A 1, A 2, B 1, B 2)$, and if $\left(O_{1}, O_{2}, O_{3}, O_{4}\right)$ is close to $(B 1, B 2, C 1, C 2)$, and if $B$ is close to $(B 2)$, then $\left(M_{1}, M_{2}, M_{3}, M_{4}\right)$ go to ( $A 1, B 2, C 1, C 2)$ ". Now if we represent the grid position by using digital coordinates, the strategy in Example 1 can be denoted as follows.

○ Mine 11122122 ;

- Oppo 21223132 ;

○ Ball 22 ;

o Dstn 11223132 .

\section{Hub StRATEgy}

Before we discuss the problem of Hub Strategy, firstly we introduce the ranking model of strategies.

K. Bryan and T. Leise [3] rank the importance of web pages according to an eigenvector of a weighted link matrix. This model is helpful to rank strategies. But strategy ranking is different to page ranking.

In [3], a core idea in assigning an importance score to any given web page is that the page's score is derived from the links made to that page from other web pages. The links to a given page are called the backlinks for that page. The web thus becomes a democracy where pages vote for the importance of other pages by linking to them. 


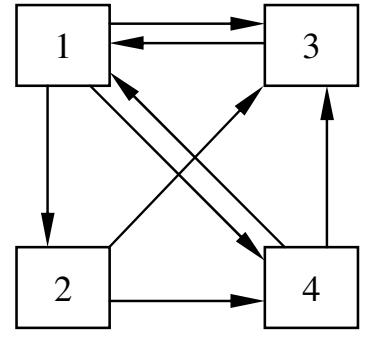

Figure 3. An example of a strategies set with only four strategies.

However, in strategy ranking, the strategy's score is derived from the links made from that strategy to other strategies. The links from a given strategy are called the forwardlinks for that strategy. The strategy thus becomes a power where strategies influence other strategies by linking to them. The strategy that has more links to other strategies is more important because that strategy has more influence on the process of game, the game situation is mostly depend on the follow-up execution of that strategy. In addition, this kind of strategy is a $h u b$ because many other strategies have to be achieved through it. If we can destroy opponent's hub strategy, then we eliminate most of the follow-up rules of opponent, and vice versa.

According to the approach presented in [3], we can obtain our method to rank strategies. Firstly suppose the strategies set of interest contains $n$ strategies, each strategy indexed by an integer $k, 1 \leq k \leq n$. A typical example is illustrated in Fig. 3, in which an arrow from strategy A to strategy B indicates a link from strategy A to strategy B. Such a strategies set is an example of a directed graph. We'll use $x_{k}$ to denote the importance score of strategy $k$ in the set. $x_{k}$ is nonnegative and $x_{j}>x_{k}$ indicates that strategy $j$ is more important than strategy $k$ (so $x_{j}=0$ indicates that strategy $j$ has the least possible importance score).

A very simple approach is to take $x_{k}$ as the number of forwardlinks for strategy $k$. In the example in Fig. 3, we have $x_{1}=3, x_{2}=2, x_{3}=1$, and $x_{4}=2$, so that strategy 1 is the most important, strategies 2 and 4 tie for second, and strategy 3 is least important. A link from strategy $k$ becomes a power for strategy $k$ 's importance.

This approach ignores an important feature one would expect a ranking algorithm to have, namely, that a link from strategy $k$ to an important strategy should boost strategy $k$ 's importance score more than a link to an unimportant strategy. For example, in the strategies set of Fig. 3, strategies 2 and 4 both have two forwardlinks: each links to the other, but strategy 4's second forwardlink is to the seemingly important strategy 1 , while strategy 2's second forwardlink is to the relatively unimportant strategy 4 . As such, perhaps we should rate strategy 4's importance higher than that of strategy 2.

As a first attempt, let's compute the importance score of strategy $j$ as the sum of the scores of all strategies linking from strategy $j$. For example, consider the strategies set of Fig. 3. The score of strategy 1 would be determined by the relation $x_{1}=x_{2}+x_{3}+x_{4}$. Since $x_{3}$ and $x_{4}$ will depend on $x_{1}$, this scheme seems strangely self-referential, but it is the approach we will use, with one more modification. We don't want a single individual to gain influence merely by sending out multiple arrows. In the same vein, we seek a scheme in which a strategy doesn't gain extra influence simply by linking to lots of other strategies. If strategy $j$ contains $n_{j}$ backlinks, one of which links from strategy $k$, then we will boost strategy $k$ 's score by $x_{j} / n_{j}$, rather than by $x_{j}$. To quantify a set of $n$ strategies, let $L_{k} \subset\{1,2, \cdots, n\}$ denote the set of strategies with a backlink from strategy $k$, that is, $L_{k}$ is the set of strategy $k$ 's forwardlinks. For each $k$ we require

$$
x_{k}=\sum_{j \in L_{k}} x_{j} / n_{j}
$$

where $n_{j}$ is the number of ingoing links to strategy $j$ (which must be positive since if $j \in L_{k}$, then strategy $j$ links from at least strategy $k$ ). We will assume that a link from a strategy to itself will not be counted, and we should delete this kind of self-loop strategy from strategies set because it's harmful to the robot's action, which would make robots be in a trap of executing repeated actions.

Fig. 3 shows an example of a strategies set with only four strategies. For strategy 1 , we have $x_{1}=x_{2} / 1+x_{3} / 3+x_{4} / 2$, since strategy 2 , strategy 3 and strategy 4 are forwardlinks for strategy 1 and strategy 2 contains only one backlink, strategy 3 assembles three backlinks, while strategy 4 receives two backlinks. Similarly, $x_{2}=x_{3} / 3+x_{4} / 2$, $x_{3}=x_{1} / 2, x_{4}=x_{1} / 2+x_{3} / 3$. These linear equations can be written as

$$
\left[\begin{array}{cccc}
0 & 1 & \frac{1}{3} & \frac{1}{2} \\
0 & 0 & \frac{1}{3} & \frac{1}{2} \\
\frac{1}{2} & 0 & 0 & 0 \\
\frac{1}{2} & 0 & \frac{1}{3} & 0
\end{array}\right]\left[\begin{array}{l}
x_{1} \\
x_{2} \\
x_{3} \\
x_{4}
\end{array}\right]=\left[\begin{array}{l}
x_{1} \\
x_{2} \\
x_{3} \\
x_{4}
\end{array}\right] .
$$

In this case we obtain $x_{1}=0.3750, x_{2}=0.1875$, $x_{3}=0.1875, x_{4}=0.2500$. Thus strategy 1 gets the highest importance score.

It should be noted that the node with highest importance score may be foible of the system. Opponent may hinder the implementation of our strategies by attacking this node, and vice versa. For example, strategy 1 is the hub strategy in Fig. 3. The strategy 2 could be fruitless if the implementation of strategy 1 is hindered. Consequently, it is necessary to decrease the influence of hub strategy to the other strategies, so that the follow-up strategies can play a role.

\section{Methodology And Experiment}

Robot soccer simulator is a good test bed for strategies set. In the simulator, two teams, such as red and blue team, 
Table I

LAST 7 STRATEGIES OF REDI.

\begin{tabular}{lllll}
\hline Rule & Mine & Oppo & Ball & Dstn \\
\hline 13 & 31344243 & 42435154 & 42 & 41435253 \\
14 & 31344243 & 42435154 & 42 & 42435253 \\
15 & 31344243 & 42435154 & 42 & 32435253 \\
16 & 41435253 & 52536263 & 52 & 52536263 \\
17 & 42435253 & 52536263 & 52 & 52536263 \\
18 & 32435253 & 52536263 & 52 & 42536263 \\
19 & 21243233 & 42435154 & 33 & 31344243 \\
\hline
\end{tabular}

Table II

LAST 6 STRATEGIES OF REDII.

\begin{tabular}{lllll}
\hline Rule & Mine & Oppo & Ball & Dstn \\
\hline 13 & 31344243 & 42435154 & 42 & 41435253 \\
14 & 32334243 & 42435154 & 42 & 42435253 \\
15 & 22324243 & 42435154 & 42 & 32435253 \\
16 & 41435253 & 52536263 & 52 & 52536263 \\
17 & 42435253 & 52536263 & 52 & 52536263 \\
18 & 32435253 & 52536263 & 52 & 42536263 \\
\hline
\end{tabular}

Table III

LAst 6 StRategies OF BLUE.

\begin{tabular}{lllll}
\hline Rule & Mine & Oppo & Ball & Dstn \\
\hline 13 & 32332223 & 41443233 & 32 & 42432233 \\
14 & 42432233 & 52534243 & 42 & 52533243 \\
15 & 52533243 & 52534243 & 52 & 62634253 \\
16 & 31323323 & 41443233 & 32 & 41424322 \\
17 & 41424322 & 52534243 & 42 & 51525332 \\
18 & 51525332 & 62635253 & 52 & 62635242 \\
\hline
\end{tabular}

will share the same strategies selection algorithm, prediction of movement, tactics, and so on, which would eliminate the impact of the simulator, and distinctly display differences between the two strategies sets. According to the game simulation result, it's easy to know which strategies set is better, because the better strategies set could achieve better competition result.

In our method, firstly we detect the hub strategy based on the ranking model presented in Section III, then we replace the hub strategy by other new strategies in order to decrease the influence of hub strategy to follow-up strategies. In our experiment, there are three strategies set, named RedI, RedII and Blue, where RedI contains 19 strategies, RedII and Blue contain 18 ones. The last 7 strategies of RedI are displayed in Table I, and last 6 strategies of RedII and Blue in Table II and Table III respectively. These strategies are the difference of the three strategies set.

Fig. 4 corresponds to the structure of last 7 strategies in RedI, and Fig. 5 shows the structure of last 6 strategies in RedII. Apparently, in RedI strategy 19 is a hub strategy, while in RedII they are parallel strategies. Table IV lists the common 12 strategies of all three strategies set.

Table V lists ten games' results of RedI vs Blue, where RedI team got three wins, three defeats and four ties. Ta-

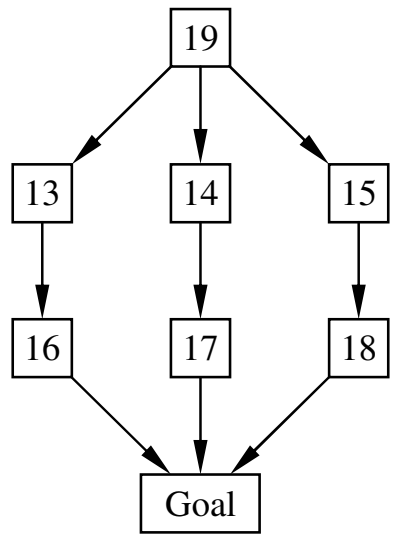

Figure 4. Graph of Last 7 strategies in RedI.

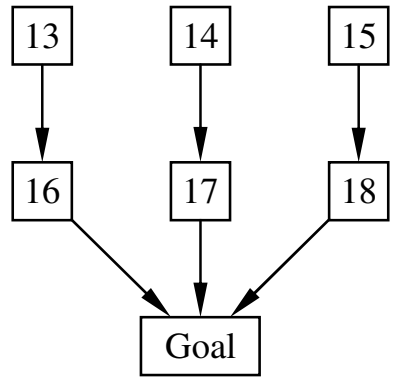

Figure 5. Graph of Last 6 strategies in RedII.

ble VI lists ten games' results of RedII vs Blue, where RedII team got four wins, two defeats and four ties. Obviously, the strategies set of RedII team is better than RedI. The reason is that strategies set Blue hinders the implementation of hub strategy 19 in RedI, while in RedII there is not hub strategy and all strategic implementation are resultful. Therefore, the problem of hub strategy could be resolved by replacing hub strategy by parallel strategies.

\section{CONClusion}

Description of strategy is an open question. Many researchers propose different approaches to describe strategies. We extract the concept of strategy grid among these approaches, and apply it to the strategies description. The description of strategies in our work is pithy, the game simulation testify its validity.

In this paper, we also present a method to improve the hub strategy in robot soccer game. We test the method in simulation game, because in the simulator everything is same for two teams except strategies, which can eliminate the impact of external factors. The differences of the game results simply reflect the performance of strategies set. We firstly detect the hub strategy by ranking model proposed in this paper, then replace the hub strategy by parallel strategies. Now we get a new strategies set RedII. If the game result between RedII and Blue is better than that of RedI and 
Table IV

The 12 Common Strategies of Three Strategies Set.

\begin{tabular}{lllll}
\hline Rule & Mine & Oppo & Ball & Dstn \\
\hline 01 & 32332124 & 42435154 & 43 & 42433133 \\
02 & 42433133 & 42335253 & 42 & 52533243 \\
03 & 52533243 & 52435354 & 53 & 62633353 \\
04 & 31433223 & 52435342 & 31 & 41534233 \\
05 & 41534233 & 51425241 & 41 & 51524143 \\
06 & 51524243 & 51425241 & 42 & 52625253 \\
07 & 52524253 & 51525252 & 52 & 62534363 \\
08 & 42433233 & 33444342 & 43 & 52534243 \\
09 & 23232223 & 23343332 & 33 & 32332223 \\
10 & 22231233 & 13233332 & 23 & 23131223 \\
11 & 52531143 & 11435342 & 12 & 32331232 \\
12 & 32331232 & 11435342 & 12 & 23232232 \\
\hline
\end{tabular}

Table V

REDI VS BLUE.

\begin{tabular}{lllllllllll}
\hline Team & \multicolumn{11}{c}{ Score } \\
\hline RedI & 1 & 0 & 1 & 2 & 0 & 0 & 1 & 2 & 3 & 1 \\
Blue & 1 & 0 & 1 & 0 & 1 & 0 & 0 & 0 & 4 & 3 \\
\hline
\end{tabular}

Table VI

REDII VS BLUE.

\begin{tabular}{lllllllllll}
\hline Team & \multicolumn{11}{c}{ Score } \\
\hline RedII & 1 & 0 & 1 & 2 & 1 & 1 & 1 & 0 & 2 & 0 \\
Blue & 0 & 1 & 0 & 3 & 1 & 1 & 0 & 0 & 1 & 0 \\
\hline
\end{tabular}

Blue on the statistical level, we can say the new strategies set RedII is better than RedI, and also we can say the method to improve hub strategy is effective. Our experiment prove the validity of our method. In our experiment, we eliminate the impact of external factors at mostly. In the strategies set, only few strategies is different, which means the difference of strategies set just focuses on hub strategy, therefore the experiment result is representative.

\section{REFERENCES}

[1] A. Bezek. Discovering strategic multi-agent behavior in a robotic soccer domain. In Proceedings of the fourth international joint conference on Autonomous agents and multiagent systems, pages 1177-1178. ACM, 2005.

[2] A. Bezek, M. Gams, and I. Bratko. Multi-agent strategic modeling in a robotic soccer domain. In Proceedings of the fifth international joint conference on Autonomous agents and multiagent systems, pages 457-464. ACM, 2006.

[3] K. Bryan and T. Leise. The $\$ 25,000,000,000$ eigenvector: The linear algebra behind google. SIAM Review, 48(3):569-581, 2006.

[4] M. Campbell, A.J. Hoane, et al. Deep blue. Artificial Intelligence, 134(1-2):57-83, 2002.

[5] H.P. Huang and C.C. Liang. Strategy-based decision making of a soccer robot system using a real-time self-organizing fuzzy decision tree. Fuzzy Sets and Systems, 127(1):49-64, 2002.
[6] J. Martinovič, V. Snášel, E. Ochodková, L Zołtá, J. Wu, and A. Abraham. Robot soccer - strategy description and game analysis. In Modelling and Simulation, 24th European Conference ECMS 2010, Kuala Lumpur, Malaysia, June 2010.

[7] R. Ros, J.L. Arcos, R. Lopez de Mantaras, and M. Veloso. A case-based approach for coordinated action selection in robot soccer. Artificial Intelligence, 173(9-10):1014-1039, 2009.

[8] J. Schaeffer, N. Burch, Y. Björnsson, A. Kishimoto, M. Müller, R. Lake, P. Lu, and S. Sutphen. Checkers is solved. Science, 317(5844):1518, 2007.

[9] J. Wu, V. Snášel, J. Martinovič, E. Ochodková, and A. Abraham. Loop strategies and application of rough set theory in robot soccer game. In Soft Computing Models in Industrial and Environmental Applications, 6th International Conference SOCO 2011, pages 117-125, Spain, Apr. 2011. Springer. 